Webley, D. M., Duff, R. B. \& F ARMer, V. C. (1958). J. gen. Microbiol. 18, 733-746

\title{
The Influence of Chemical Structure on $\beta$-oxidation by Soil Nocardias
}

\author{
By D. M. WEBLEY, R. B. DUFF AND V. C. FARMER \\ The Macaulay Institute for Soil Research, Craigiebuckler, Aberdeen
}

\begin{abstract}
SUMMARY: Studies of the $\beta$-oxidation of the fatty acid side-chain of certain $\omega$-aryland $\omega$-aryloxy- $n$-alkylcarboxylic acids by Nocardia opaca (strain $\mathrm{T}_{16}$ ) and Nocardia sp. (strain $P_{2}$ ) show that $\gamma$-phenylbutyric is more rapidly oxidized than $\gamma$-phenoxybutyric acid. The effect of the oxygen bridge is even more striking when $\gamma$-(1-naphthyl)- and $\gamma$-(1-naphthyloxy)-butyric acids are compared. The rate of $\beta$-oxidation (by strain $\mathrm{T}_{16}$ ) of 3-, 4-, and 2-isomers decreases in that order. This applies to monochloro- and monomethylphenoxybutyric acids and to monochlorophenoxypropionic acids. Substitution in position 2 has by far the greatest effect and chlorine exerts a bigger influence than a methyl group in all positions. The conversion of $\gamma$-(2-methyl4-chlorophenoxy)-butyric acid (MCPB) and $\gamma$-(2:4-dichlorophenoxy)-butyric acid (2:4-DB) is very slow and proceeds only to $\beta$-hydroxy acids. On the other hand, $\epsilon$-(2-methyl-4-chlorophenoxy)-, $\epsilon$-(2:4-dichlorophenoxy)- and $\epsilon$-(2-chlorophenoxy)caproic acids are relatively rapidly converted to their corresponding butyric acid derivatives. With strain $T_{16} \omega$-(2-naphthyloxy)-butyric and propionic acids are more rapidly converted to their corresponding acetic acids and phenols respectively than the $\omega$-(1-naphthyloxy) compounds. The rate of $\beta$-oxidation of $\gamma$-phenyl-, $\gamma$-(3-indolyl)and $\gamma$-(1-naphthyl)- butyric acids (by strain $P_{2}$ ) decreases in that order. It has been shown that $\beta$-hydroxy acid intermediates are formed from $\omega$-aryloxybutyric acids and those from MCPB, $\gamma$-(2-methyl-4-chlorophenoxy)-crotonic and $\gamma$-(2-naphthyloxy)-butyric acids have been isolated and identified.
\end{abstract}

It was found (Webley, Duff \& Farmer, 1957) that $\beta$-hydroxy acid intermediates accumulated during the conversion of 3- and 4-chlorophenoxybutyric acids to their corresponding acetate derivatives by washed suspensions of Nocardia opaca. Similarly, it was found (Webley, Duff \& Farmer, 1955, 1956) that the breakdown of the side-chain of $\beta$-phenylpropionic acid and of $\alpha$-(1naphthyl)-hendecane gave $\alpha \beta$-unsaturated acids as intermediates. The coenzyme $A$ derivatives (Fig. 1 ) of $\beta$-hydroxy and $\alpha \beta$-unsaturated acids are known intermediates in $\beta$-oxidation (Lynen, 1953; Mahler, 1953), but the free acids had not been previously detected. It seemed therefore that their production during $\beta$-oxidation by $N$. opaca of the compounds mentioned above was associated with $\omega$-aryl and $\omega$-aryloxy substitution. In the present work with $N$. opaca and an unidentified soil nocardia it has been found that the ring structure, the oxygen bridge and the position and nature of the ring substitutents affect the rate of $\beta$-oxidation and the accumulation of intermediates when the side-chain of the metabolite is short. Other $\beta$-hydroxy acids now identified include those from $\gamma$-(2-methyl-4-chlorophenoxy)-butyric acid (MCPB) and $\gamma$-(2-naphthyloxy)-butyric acid. 


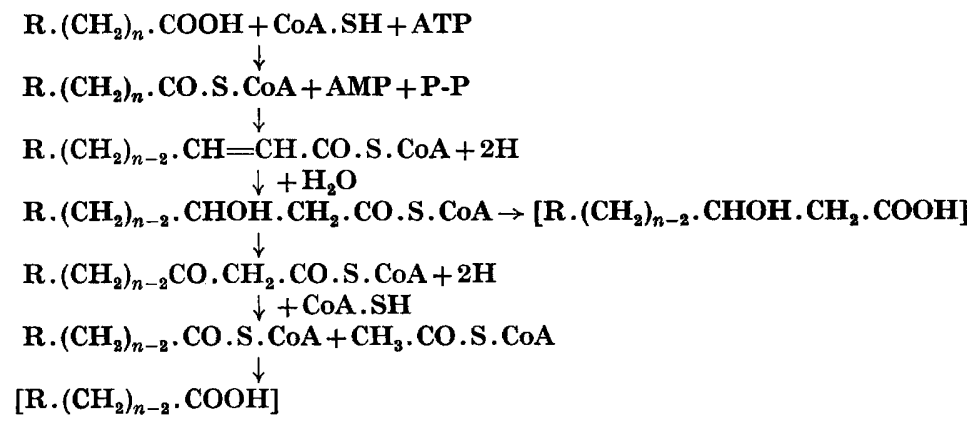

Fig. 1. Stages in the $\beta$-oxidation of substituted fatty acids. ATP=Adenosine triphosphate; $\mathbf{A M P}=$ adenosine monophosphate; $\mathbf{P}-\mathbf{P}=$ pyrophosphate $; \mathbf{C o A} . \mathbf{S H}=$ coenzyme A (reduced). In the present work $n=5,3$ or 2 to give the corresponding substituted caproic, butyric or propionic acids and $\mathbf{R}=$ various aryl or aryloxy groups. Products isolated are indicated thus [ ].

\section{METHODS}

Organisms. Nocardia opaca strain $\mathrm{T}_{16}$ (Webley, 1954) and Nocardia sp. strain $\mathbf{P}_{2}$ isolated from soil by Dr Treccani (University of Milan) were used throughout.

Production of organisms. Strain $\mathrm{T}_{\mathbf{1 6}}$ was grown as described previously (Webley et al. 1955). In some experiments $1 \%(\mathrm{w} / \mathrm{v})$ phenylacetic acid (sodium salt) was used in place of glucose, $0.1 \%(\mathrm{w} / \mathrm{v})$ yeast extract (Difco) was added and the cultures were incubated for $7-12$ days at $25^{\circ}$. Strain $P_{2}$ was grown similarly, but $1 \%(\mathrm{w} / \mathrm{v})$ glucose nutrient broth was used and the flasks were incubated for 14 days. The organisms were harvested with aseptic precautions for the large-scale incubation experiments.

Manometric experiments. Measurements of $\mathrm{O}_{2}$ uptake were carried out as already described (Webley et al. 1955), substrates being tipped in from the side arm of the Warburg flasks after the overnight incubation required to decrease endogenous activity.

Large-scale experiments. These were carried out as in previous work (Webley, et. al. 1955). The flasks contained 25-100 ml. of 0.0133 $\mathrm{M}$-phosphate buffer (pH 7·2), 0.004 M (unless otherwise stated) $\omega$-aryl- or $\omega$-aryloxy-alkylcarboxylic acid (sodium salt) and the equivalent of $200-800 \mathrm{mg}$. dry weight of washed glucose-grown organisms. The volume was usually determined by the amount of the substrate available. Organisms were added aseptically to the sterile substrate buffer mixture. When comparing the rate of conversion of different compounds equal samples of the same suspension were added to each flask. It was necessary in certain experiments to add fresh organisms at intervals of 7 days to bring about sufficient conversion of the substrates for chromatographic and spectrochemical analysis. These experiments were terminated 7 days after the final addition of fresh organisms. Since this type of experiment was often continued for several weeks strict aseptic technique was necessary. The contents of all flasks were examined microscopically and by 
streaking on nutrient agar plates after the removal of samples, at the end of the experiments, and before addition of fresh organisms in the longer incubation experiments. Any flask which became contaminated was discarded. Samples (5-25 ml.) or the whole contents of the flasks were taken when required and after removal of the organisms the supernatant fluids were acidified and extracted with ether. The ether-soluble products were subjected to chromatographic and spectrochemical analysis as described below. Sometimes the supernatant fluids were examined directly.

Spectrochemical techniques. Where the product was a single substance, it was identified from its infrared spectrum in the solid state, such spectra being highly specific, sensitive even to crystalline form. Mixed products were examined in dioxan solution $5 \%(\mathrm{w} / \mathrm{v})$ in $0.1 \mathrm{ml}$. optical cells since in this solvent interaction between the components of a mixture is avoided, as each component associates preferentially with solvent molecules. Under these conditions the $\beta$-hydroxy acid intermediates, the substituted acrylic acids, and the aryloxyacetic acids are each distinguished from other components by specific absorption bands. The alcoholic hydroxyl group of the $\beta$-hydroxy acid intermediates absorbs at $3420 \mathrm{~cm} .^{-1}$ in dioxan solution, well separated from the absorption of acidic hydroxyl groups at $3070 \mathrm{~cm} .^{-1}$, as shown in Fig. 2, for $\beta$-hydroxy- $\gamma$-(2-methyl-4-chlorophenoxy)-butyric acid. Estimates of concentration of $\beta$-hydroxy acid in mixtures were made by comparison with standards, the limit of detectability being about $10 \%$ of the total acids.

Similarly, the infrared absorption band due to the $\mathrm{C}=\mathrm{C}$ bond at $1666 \mathrm{~cm} .^{-1}$ distinguished the acrylic acid intermediates from the other components (Fig. 3). About $5 \%$ of $\gamma$-(2-methyl-4-chlorophenoxy)-crotonic acid was just detectable with certainty in mixtures with $\gamma$-(2-methyl-4-chlorophenoxy)butyric acid, and a similar sensitivity is to be expected for other phenoxycrotonic acids. The carbonyl group of the aryloxyacetic acids (seven examples were examined) showed doublet absorption at 1770 and $1745 \mathrm{~cm} \cdot .^{-1}$ in dioxan solution of which the higher frequency component is not given by either the aryloxybutyric or the $\beta$-hydroxybutyric acids (Fig. 3). This band permitted the estimation of phenoxyacetic acids down to $10 \%$ in mixtures. The higher frequency component probably originates from a rotational isomer of the phenoxyacetic acid complex with dioxan, in which the oxygen of the carbonyl group is closest to the ether oxygen of the phenoxy group, whilst the lower frequency component corresponds to other rotational isomers in which interaction between the two oxygen atoms is slight. Similar effects have been noted when a halogen atom is in the $\alpha$-position to a carbonyl group (Bellamy, Thomas \& Williams, 1956), the carbonyl frequency being abnormally high only when the halogen is close in space to the oxygen atom.

Ultraviolet absorption spectrometry was used to follow the conversion of $\omega$-aryloxypropionic acids to the corresponding phenols, by measurements on the supernatants of samples withdrawn from large-scale experiments. The total concentration of the aryloxy group was estimated by measurements in acid solution, in which the absorption of the phenols closely resembles the parent aryloxypropionic acids. In alkaline solution, the phenols give the 
intense distinctive absorption of their anions, whilst the spectra of the aryloxypropionates show negligible changes. Accordingly, the phenols formed were estimated from the change in absorption on going from acid to alkaline solution, at $290 \mathrm{~m} \mu$. for 3- and 4-chlorophenol, at $240 \mathrm{~m} \mu$. for 2-naphthol, and at $245 \mathrm{~m} \mu$. for 1-naphthol.

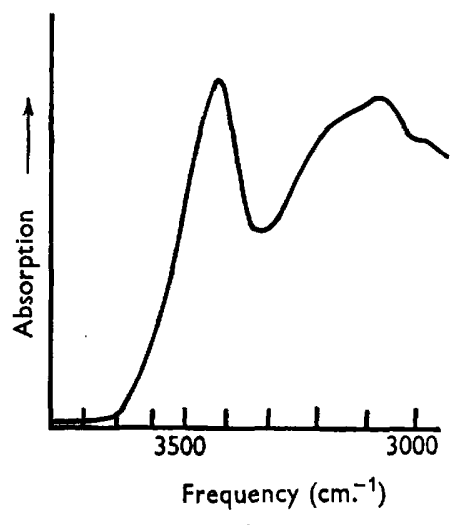

Fig. 2

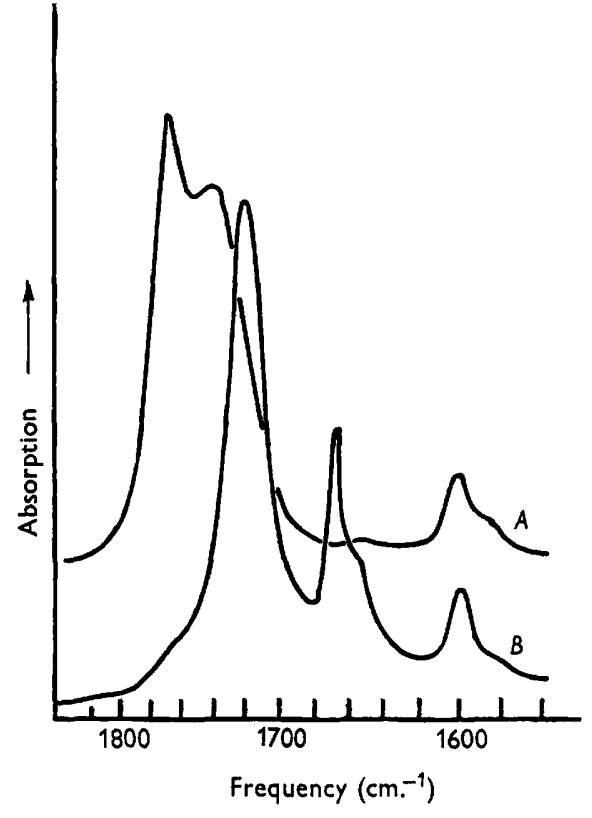

Fig. 8

Fig. 2. Absorption due to $\mathrm{O}-\mathrm{H}$ stretching vibrations of $\beta$-hydroxy- $\gamma$-(2-methyl-4-chlorophenoxy)-butyric acid.

Fig. 3. Absorption due to $\mathrm{C}=\mathrm{O}$ and $\mathrm{C}=\mathrm{C}$ stretching vibrations of $\mathrm{A}, 2$-methyl-4-chlorophenoxyacetic acid, and $B, \gamma$-(2-methyl-4-chlorophenoxy)-crotonic acid.

Paper chromatography. This was carried out generally as described previously (Webley et al. 1955). Occasionally the 'multiple development' technique of Jeanes, Wise \& Dimler (1951) was used to obtain sufficient separation. It was particularly convenient with acids as it was unnecessary to use separate test strips to follow the resolution of the mixtures. The dried paper was sprayed with Universal Indicator solution $(1: 1(\mathrm{v} / \mathrm{v})$ mixture with water adjusted to $\mathrm{pH} 9.0$ with dilute sodium hydroxide) and the position of the spots noted. The paper was then developed a second or third time. The indicator did not interfere. The solvent used by Byrde, Harris \& Woodcock (1956) gave better separation of $\omega$-naphthyl and $\omega$-naphthyloxy substituted acids than the usual solvent (Fewster \& Hall, 1951). For quantitative work, samples were dissolved in ethanol and a suitable amount corresponding to about $0.8 \mathrm{mg}$. total acids applied to the chromatogram. The position of the various acids was determined by using test strips. The strips containing the individual acids were immersed directly in $0.1 \%(\mathrm{w} / \mathrm{v})$ sodium hydroxide solution (usually 
$5 \mathrm{ml}$.) for $15 \mathrm{~min}$. at $50^{\circ}$ and the decanted solution used for ultraviolet spectrometry. This technique was used to follow the $\beta$-oxidation of the chlorophenoxybutyric acids. The relative concentration of the components were estimated from their absorption $(e \div 1400)$ at 275 to $280 \mathrm{~m} \mu$. Control experiments showed that a recovery of $95 \%$ of the acid applied to the paper was to be expected.

Melting-points. These were determined with the Kofler heating stage.

\section{RESULTS}

The breakdown of the fatty acid side-chain of $\gamma$-phenyl-and $\gamma$-phenoxy-
butyric acids by nocardia strains

In contrast to phenylacetic acid, phenoxyacetic acid was not attacked by strain $\mathbf{T}_{16}$ but increases in oxygen uptake were obtained with phenoxybutyric and phenoxycaproic acids, presumably due to the breakdown of the fatty acid side-chain (Table 1). To confirm this a large-scale experiment was set up

Table 1. The oxygen uptake of Nocardia opaca strain $T_{16}$ in the presence of phenylacetic, phenoxyacetic, phenoxybutyric and phenoxycaproic acids

Each vessel contained equiv. $15.3 \mathrm{mg}$. dry weight of a washed suspension of phenylacetate-grown $N$. opaca, $0.5 \mathrm{ml}$. 0.066 M-phosphate buffer $(\mathrm{pH} 7 \cdot 2)$ and distilled water to $2 \cdot 0 \mathrm{ml}$. After overnight incubation $0.5 \mathrm{ml}$. of distilled water or $0.02 \mathrm{M}$-sodium salts of the acids were tipped from the side arm.

$\begin{array}{lc} & \mathrm{O}_{2} \text { uptake in } \\ \text { Addition } & 180 \text { min. }(\mu 1 .) \\ \text { None } & 93 \\ \text { Phenylacetate } & 984 \\ \text { Phenoxyacetate } & 112 \\ \gamma \text {-Phenoxybutyrate } & 219 \\ \epsilon \text {-Phenoxycaproate } & 558\end{array}$

with phenoxybutyrate and $\mathbf{T}_{16}$ organisms as described in 'Methods'. Samples were removed aseptically after 17, 24, 41 and $48 \mathrm{hr}$. Paper chromatography of the extracts showed two spots in the early samples $(17,24$ and $41 \mathrm{hr}$.) one corresponding to the original material. The latter had disappeared in the $48 \mathrm{hr}$. sample leaving only a substance with the $\boldsymbol{R}_{F}$ of phenoxyacetic acid. Material from the $41 \mathrm{hr}$. sample, dissolved in dioxan, showed the presence of both a $\beta$-hydroxy acid (about $30 \%$ ) and phenoxyacetic acid (about $25 \%$ ) by infrared analysis. It seems that the $\beta$-hydroxy acid formed from phenoxybutyric acid did not separate from phenoxyacetic acid on the paper chromatograms in contrast to the $\beta$-hydroxy acid formed from 3- and 4-chlorophenoxybutyric acid (Webley et al. 1957). Chromatographic examination of the samples failed to reveal the presence of phenols or phenolic acids.

The rates of breakdown of phenoxy- and phenylbutyric acids were compared with strain $\mathbf{P}_{2}$, which does not attack phenylacetic acid (Webley et al. 1956). A preliminary manometric experiment suggested that the side-chain of phenylbutyric acid was broken down more easily than that of phenoxybutyric acid. This was confirmed by an experiment in which samples were 
removed after 24, 48, 65, $120 \mathrm{hr}$. Paper chromatography and subsequent examinations of the infrared spectra showed that phenylbutyric acid had been completely converted to phenylacetic acid by $24 \mathrm{hr}$. With phenoxybutyric acid conversion to a $\beta$-hydroxy acid $(20 \%)$ and phenoxyacetic acid $(40 \%)$ was found after $65 \mathrm{hr}$. (infrared spectra in dioxan). In a further experiment with $0.02 \mathrm{M}$-phenylbutyrate and strain $\mathbf{P}_{2}$ organisms samples were taken at $8,21,31$ and $48 \mathrm{hr}$. Those showing about $50 \%$ conversion by paper chromatography (8 and $21 \mathrm{hr}$.) were subjected to infrared analysis. No $\beta$-hydroxy acid absorption was detected in dioxan solution, indicating that this intermediate amounted to less than $10 \%$ of the total acids.

\section{, \\ The metabolism of the fatty acid side-chain of ring-substituted $\omega$-(phenoxy)-n-alkylcarboxylic acids by nocardia strains}

Monochlorophenoxybutyric and caproic acids. Table 2 shows the oxygen uptake of strain $\mathbf{T}_{16}$ with phenoxybutyric acid and its 2-, 3- and 4-monochloro derivatives. Substitution in the 2-position brings about a marked reduction. A larger scale experiment was next set up with two sets of flasks containing 2-, 3- or 4-monochlorophenoxybutyric acids (sodium salts) and equal samples from a washed suspension of $T_{16}$ organisms. After 7 days of incubation one set was examined by paper chromatography and infrared analysis. The 3and 4-monochlorophenoxybutyric acids had been completely converted whilst $75 \%$ of the 2-chloro compound remained unchanged. After 14 days of incubation examination of the other set revealed that there was still $67 \%$ of the 2-chlorophenoxybutyric acid present. It was shown that these differences were not simply a reflection of the relative strengths of the acids (based on the $\mathrm{pH}$ value of half-neutralized equimolar solution of the acids in $50 \%$ aqueous ethanol).

Table 2. The oxygen uptake of Nocardia opaca strain $T_{16}$ in the presence of phenoxybutyric and 2-, 3-, and 4-monochlorophenoxybutyric acids

Each vessel contained equiv. $7 \cdot 1 \mathrm{mg}$. dry weight of a washed suspension of glucose-grown N. opaca, $0.5 \mathrm{ml} .0 .066 \mathrm{M}$-phosphate buffer $(\mathrm{pH} 7 \cdot 2)$ and distilled water to give $2.0 \mathrm{ml}$. After overnight incubation $0.5 \mathrm{ml}$. of distilled water or $0.05 \mathrm{M}$-sodium salts of the acids was tipped in from the side arm.

$\begin{array}{lc} & \mathrm{O}_{2} \text { uptake in } 180 \mathrm{~min} \\ & (\mu \mathrm{l} .) \\ \text { None } & 59 \\ \gamma \text {-Phenoxybutyrate } & 121 \\ \gamma \text {-(2-Chlorophenoxy)-butyrate } & 82 \\ \gamma \text {-(3-Chlorophenoxy)-butyrate } & 118 \\ \gamma \text {-(4-Chlorophenoxy)-butyrate } & 124\end{array}$

The rates of conversion by $T_{16}$ organisms of the 3 - and 4-chloro compounds were also compared (Table 3 ). The 3-chloro compound was converted at a somewhat faster rate than the 4-chloro compound. The $\beta$-hydroxy acid intermediates formed during the conversion of these compounds have already been identified (Webley et al. 1957). 
Comparison of the oxidation of 2-chlorophenoxycaproic and 2-chlorophenoxybutyric acids showed that only the former compound was actively metabolised. In a large-scale experiment paper chromatography showed that 2-chlorophenoxycaproic acid had been completely converted within $65 \mathrm{hr}$. to a substance with an $R_{p}$ corresponding to 2-chlorophenoxybutyric acid while 2-chlorophenoxybutyric acid had remained unchanged.

\section{Table 3. The rate of conversion of 3- and 4- monochlorophenoxybutyric acids by Nocardia opaca strain $T_{16}$}

Flasks contained $0 \cdot 0133 \mathrm{~m}$-phosphate buffer $(\mathrm{pH} \mathrm{7 \cdot 2)}$ and $0.004 \mathrm{M}-3$ - or 4-monochlorophenoxybutyric acids (as sodium salt) and equiv. $231.6 \mathrm{mg}$. dry weight glucose-grown $N$. opaca to give final volume of $50 \mathrm{ml}$. Flasks were incubated on shaking machine at $25^{\circ}$, 25 ml. suspension removed aseptically after 3 days, and the remainder after 5 days. After centrifugation the supernatant fluids were extracted and quantitatively spotted on paper chromatograms. The acids were eluted separately and their concentration estimated from their absorption maxima $(e \doteqdot 1400)$ at $275-280 \mathrm{~m} \mu$.

\begin{tabular}{|c|c|c|c|c|}
\hline & \multicolumn{3}{|c|}{ Percentage of total products } \\
\hline & & $\begin{array}{l}\text { Butyrate } \\
\text { derivative }\end{array}$ & $\begin{array}{c}\beta \text {-Hydroxy } \\
\text { acid } \\
\text { intermediate }\end{array}$ & $\begin{array}{c}\text { Acetate } \\
\text { derivative }\end{array}$ \\
\hline $\left.\begin{array}{l}\gamma \text {-(3-Chlorophenoxy)-butyrate } \\
\gamma \text {-(4-Chlorophenoxy)-butyrate }\end{array}\right\}$ & 3 days & $\left\{\begin{array}{l}40 \cdot 5 \\
77\end{array}\right.$ & $\begin{array}{l}59 \cdot 5 \\
23\end{array}$ & - \\
\hline $\left.\begin{array}{l}\gamma \text {-(3-Chlorophenoxy)-butyrate } \\
\gamma \text {-(4-Chlorophenoxy)-butyrate }\end{array}\right\}$ & 5 days & $\left\{\begin{array}{l}22 \\
51\end{array}\right.$ & $\begin{array}{l}67 \\
49\end{array}$ & 11 \\
\hline
\end{tabular}

Monochlorophenoxypropionic acids. The $\beta$-oxidation of phenoxypropionic acids might be expected to yield phenols (by way of the unstable phenylcarbonates, $\mathrm{C}_{6} \mathrm{H}_{5} . \mathrm{O} \cdot \mathrm{CH}_{2} \cdot \mathrm{CH}_{2} . \mathrm{COOH} \rightarrow \mathrm{C}_{6} \mathrm{H}_{5} . \mathrm{O} \cdot \mathrm{CO} \cdot \mathrm{OH} \rightarrow \mathrm{C}_{6} \mathrm{H}_{5} \cdot \mathrm{OH}+\mathrm{CO}_{2}$ ), and Fawcett, Ingram \& Wain (1954) showed that plant tissue can in fact convert such compounds to phenols. The side-chain breakdown of the three monochlorophenoxypropionic acids by $T_{16}$ organisms was followed by ultraviolet absorptiometry. Warburg flasks were set up containing $0.0133 \mathrm{M}$ -

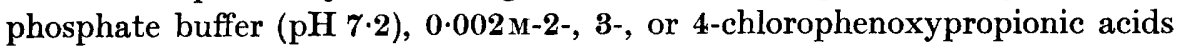
(sodium salts), and the equivalent of $51.0 \mathrm{mg}$. dry weight of glucose-grown $\mathrm{T}_{16}$ organisms to give a final volume of $2.5 \mathrm{ml}$. in each vessel. After incubation at $25^{\circ}$ for $18 \mathrm{hr}$., the flask contents were centrifuged and the supernatant liquid examined. No phenols were formed from 2-chlorophenoxypropionate, but $70 \%$ of the 3 -chloro- and $40 \%$ of the 3-chlorophenoxypropionates had been converted to phenols, whose absorption agreed with the expected products. No attack on the benzene ring occurred under these conditions, but in experiments continued over long periods there were indications of the partial conversion of the 4-chlorophenol to polyhydroxyphenols, with absorption similar to 4-chlorocatechol. These results parallel closely those obtained from the monochlorophenoxybutyric acids.

Monomethylphenoxybutyric acids. The monomethylphenoxybutyric acids had a similar effect on the oxygen uptake of $\mathbf{T}_{16}$ organisms to the monochlorophenoxybutyric acids (Table 2). A large-scale experiment was set up with 
flasks containing 2-, 3-, or 4-monomethylphenoxybutyric acids (sodium salts) and $T_{16}$ organisms. After 3 days of incubation samples from each flask were examined by paper chromatography; there had been virtually complete removal of the 3- and 4-methyl compounds but very little conversion of the 2-methyl compound. The infrared spectra of the solid products showed the formation of the acetate derivative from the 3- and 4-methylphenoxybutyric acids, although the yield from the latter was less than theoretical. Fresh organisms were added to each flask. The infrared spectra of the products at

Table 4. Comparison of products from 3-methyl-, 3-chloro-, and unsubstitutedphenoxybutyric acids during $\beta$-oxidation by Nocardia opaca strain $T_{16}$

Flasks contained 0.0133 $\mathrm{M}$-phosphate buffer $(\mathrm{pH} \mathrm{7 \cdot 2)}$ and $0 \cdot 004 \mathrm{M}-3$-methyl- or 3-chloroor unsubstituted-phenoxybutyric acids (sodium salts) and equiv. $374 \mathrm{mg}$. dry weight glucose-grown $N$. opaca to give a final volume of $100 \mathrm{ml}$. Flasks incubated at $25^{\circ}$ for $42 \mathrm{hr}$. The ether-soluble products were examined in dioxan solution by infrared spectroscopy.

$\begin{array}{lccc} & \overbrace{}^{\beta \text {-Hydroxy acids }} & \begin{array}{c}\text { Substituted } \\ \text { phenoxyacetic acid }\end{array} \\ \gamma \text {-Phenoxybutyrate } & 45 & 25 \\ \gamma \text {-(3-Methylphenoxy)-butyrate } & 35 & 20 \\ \gamma \text {-(3-Chlorophenoxy)-butyrate } & 50 & 10\end{array}$

the end of the experiment showed that the 3- and 4-methylphenoxybutyric acids had now been completely converted to the corresponding acetate derivatives. The extracted material was recrystallized (twice) from benzene. The products from 3- and 4-methylphenoxybutyric acids had m.p. $104^{\circ}$ and $139^{\circ}$, respectively, unchanged on admixture with the corresponding authentic acetates. The spectrum of the solid product from 2-methylphenoxybutyric acid indicated that the butyrate was largely unchanged.

A manometric experiment suggested that a 2-chloro- had a greater effect than a 2-methyl-substituent on the $\beta$-oxidation of phenoxybutyric acid by Nocardia opaca. Confirmation of this was obtained by means of a large-scale experiment. Since the rate of conversion of the 2-substituted compounds is very slow it was necessary to make several additions of fresh organisms. Two culture flasks were set up containing 2-chlorophenoxybutyric or 2-methylphenoxybutyric acids and $T_{16}$ organisms. Three further additions of fresh organisms were made to each flask. At the end of the experiment the infrared spectra of the products in dioxan showed that the 2-methyl- and 2-chlorophenoxybutyric acids had given $40 \%$ and $15 \%$ of the corresponding acetates, respectively; both samples contained about $30 \% \beta$-hydroxy acids. The results of a comparison of 3-methyl-, 3-chloro-phenoxybutyric acids, and the unsubstituted acid are given in Table 4. They indicate that the rate of conversion of the $\beta$-hydroxy intermediate is slower with the chlorine-substituted material. A comparison of 4-methyl- with 4-chloro-phenoxybutyric acids gave similar results. 
The effect of di-substitution of the ring on the metabolism of the fatty acid side-chain of $\omega$-phenoxyalkylcarboxylic acids by Nocardia opaca

Since substitution in position 2 (by methyl or chlorine) exerted such a marked effect on the rate of $\beta$-oxidation of the butyric acid side-chain of monosubstituted phenoxybutyric acids a few di-substituted compounds were tested. 2-Methyl-4-chlorophenoxybutyric acid (MCPB) and 2:4-dichlorophenoxybutyric acid (2:4-BD) are of special interest because of their practical importance as selective weed killers. It is known (Wain, 1956) that the herbicidal action of these substances is due to $\beta$-oxidation of the fatty acids side-chain within the plant to the corresponding acetate derivatives (MCPA or 2:4-DA) which are recognized herbicides. Flasks were set up with $T_{16}$ organisms to compare 2-methylphenoxybutyric acid with MCPB. Two further additions of organisms were made and at the end of the experiment paper chromatography showed that there had been a much greater conversion of the 2-methyl compound than of the MCPB. Infrared analysis, in dioxan, showed $30 \%$ and $15 \% \beta$-hydroxy acid intermediate from 2-methylphenoxybutyric acid and MCPB, respectively. The former sample contained $20 \%$ of the corresponding acetate derivative and the latter none. Similarly, when 3-chloro- and 4-chlorophenoxybutyric acids were compared with 3:4-dichlorophenoxybutyric acid it was found that the di-substituted compound was converted much more slowly than the mono-substituted ones. These experiments clearly demonstrated that di-substitution decreases the rate of $\beta$-oxidation. MCPB and 2:4-DB were next compared. Two flasks containing MCPB or 2:4-DB and $T_{16}$ organisms were set up in the usual way, and three further additions of fresh organisms were made. At the end of the experiment paper chromatography revealed that MCPB and 2:4-DB had been converted $50 \%$ and $20 \%$, respectively. Infrared examination of the products in dioxan solution showed that a $\beta$-hydroxy acid was produced in each case; MCPA and 2:4-DA were not detected. In a similar experiment, but with seven additions of $\mathbf{T}_{16}$ organisms chromatography showed that the original material had almost all been converted to an unknown substance. Infrared examination after recrystallization of the material from benzene showed this to be (by comparison with a synthetic specimen) the $\beta$-hydroxy derivative of MCPB. In previous work (Webley et al. 1957) it had been found possible to convert further the $\beta$-hydroxy acid formed from 3- and 4-monochlorophenoxybutyric acids to the corresponding acetate derivatives. Attempts to bring about a similar conversion of the $\beta$-hydroxy acid of MCPB were unsuccessful. To see whether 2-methyl4-chlorophenoxycrotonic acid could be converted to the $\beta$-hydroxy acid by the organism a culture flask was set up containing a $0.001 \mathrm{M}$ solution of this compound and $T_{18}$ organisms. Five further additions of fresh organisms were made. Evaporation of the ether extract obtained at the end of the experiment gave an oil, which on standing crystallized. After recrystallization from aqueous ethanol the infrared spectrum of the product agreed closely with that of authentic $\beta$-hydroxy- $\gamma$-(2-methyl-4-chlorophenoxy)-butyric acid.

In view of the powerful effect of di-substitution on the $\beta$-oxidation of the 
side-chain of phenoxybutyric acid it was thought worthwhile to see whether a similar effect operated with a longer side-chain acid. 2-Methyl-4-chlorophenoxycaproic (MCPC) and 2:4-dichlorophenoxycaproic (2:4-DC) acids gave marked increases in oxygen uptake (Table 5) when compared with MCPB and 2:4-DB, suggesting a much more rapid breakdown of the side-chain. This was confirmed in an incubation experiment with MCPC and washed $T_{16}$ organisms, where it was found that one further addition of organisms was sufficient to convert MCPC to MCPB in 3 days.

Table 5. The oxygen uptake of Nocardia opaca strain $T_{16}$ in the presence of $M C P C, M C P B, M C P A, 2: 4-D C$ and $2: 4-D B$

Each vessel contained equiv. 8.4 $\mathrm{mg}$. dry weight of a washed suspension of glucose-grown N. opaca, $0.5 \mathrm{ml}$. 0.066 M-phosphate buffer (pH 7.2) and distilled water to give $2.0 \mathrm{ml}$. After overnight incubation $0.5 \mathrm{ml}$. of distilled water or $0.005 \mathrm{M}$-sodium salts of the acids were tipped in from the side arm.

$\begin{array}{lc} & \mathrm{O}_{2} \text { uptake in } \mathbf{1 8 0} \mathrm{min} . \\ \text { Addition } & (\mu \mathrm{l} .) \\ \text { None } & 60 \\ \text { MCPC } & 210 \\ \text { MCPB } & 84 \\ \text { MCPA } & 60 \\ \text { 2:4-DC } & 189 \\ \text { 2:4-DB } & 78\end{array}$

The breakdown of the fatty acid side-chain of $\omega$-(1-naphthyl)-, $\omega$-(1-naphthyloxy)-, and $\omega$-(2-naphthyloxy)-butyric and -propionic acids by Nocardia opaca

$\gamma$-(1-naphthyloxy)- and $\gamma$-(2-naphthyloxy)-butyric acids. Byrde et al. (1956) showed that $\gamma$-(2-naphthyloxy)-butyric acid underwent $\beta$-oxidation by Aspergillus niger after hydroxylation of the naphthyl nucleus, the final product being (6-hydroxy-2-naphthyloxy)-acetic acid. They also showed that an intermediate was formed between $\gamma$-(6-hydroxy-2-naphthyloxy)- $n$-butyric acid and (6-hydroxy-2-naphthyloxy)-acetic acid but did not attempt to isolate or identify this compound. The breakdown of $\gamma$-(1-naphthyloxy)- and $\gamma$-(2-naphthyloxy)-butyric acids by the soil nocardias used here was investigated. Flasks were set up containing $0.002 \mathrm{M}$ solutions of these compounds and $\mathrm{T}_{16}$ organisms. Since only about $20 \%$ conversion of the 2-naphthyloxy compound had taken place after 8 days two further additions of fresh organisms were made. Ether extracts from samples taken immediately before the last addition showed that the product from $\gamma$-(2-naphthyloxy)-butyric acid was principally the $\beta$-hydroxy acid intermediate, by comparison of its infrared spectra with that of an authentic specimen, prepared from the corresponding ester by the method of Grün (1925). Seven days after the third addition of organisms the $\gamma$-(2-naphthyloxy)-butyric acid was found to be completely converted to the corresponding acetate (m.p. and mixed m.p. $154^{\circ}$ after two recrystallizations from benzene). In contrast, $\gamma$-(1-naphthyloxy)-butyric acid was unchanged throughout a similar experiment. Phenols or phenolic acids could not be detected in either case, showing that Nocardia opaca, strain $\mathbf{T}_{16}$, unlike $A$. niger cannot hydroxylate the naphthyl nucleus. 
$\beta$-(1-naphthyloxy)- and $\beta$-(2-naphthyloxy)-propionic acids. When the sidechains of the above compounds are metabolized by $\beta$-oxidation the expected products would be 1-naphthol and 2-naphthol respectively (cf. monochlorophenoxypropionic acids). The following experiment was carried out to follow the production (if any) of phenols. Two flasks containing $0.001 \mathrm{M}-\beta$-(1-naphthyloxy)- or $\beta$-(2-naphthyloxy)-propionic acids (sodium salts) and $\mathbf{T}_{16}$ organisms were set up and sampled after $0,4,6$ and 8 days. Ultraviolet spectrometry after removal of organisms showed that $38 \%$ of the $\beta$-(2-naphthyloxy)propionic acid was converted to 2-naphthol in 6 days, with no further change in 8 days. No naphthol was formed from $\beta$-(1-naphthyloxy)-propionic acid and in neither case was there any attack on the ring. After 12 days the experiment was ended and the extracted products examined by paper chromatography. The extract from the 2-naphthyloxy compound contained a substance with an $\boldsymbol{R}_{F}$ value corresponding to 2-naphthol. No phenols or phenolic acids were present in the material from the 1-naphthyloxy experiment. The results of this experiment parallel the previous observations with $\gamma$-(1-naphthyloxy)- and $\gamma$-(2-naphthyloxy)-butyric acids, showing the marked difference in the $\beta$-oxidation of the fatty acid side-chain depending on whether it is attached to position 1 or position 2 of the naphthyl nucleus through an oxygen bridge.

$\gamma$-(1-naphthyl)- and $\gamma$-(1-naphthyloxy)-butyric acids. Phenylbutyric acid was metabolized more rapidly than phenoxybutyric acid (see Table 2), but the difference between 1-naphthyl- and 1-naphthyloxy-butyric is even more striking as was demonstrated in the following experiment. $T_{16}$ organisms were added to two flasks containing $0.002 \mathrm{M} \gamma$-(1-naphthyl)- or $\gamma$-(1-naphthyloxy)butyrate. Analysis of samples taken after 7 days showed that 1-naphthylbutyric acid had been completely converted to the acetate, whereas the 1-naphthyloxybutyric acid was unchanged. Further addition of organisms had no effect on the latter compound.

The breakdown of the fatty acid side-chain of $\gamma$-phenyl-, $\gamma$-(3-indolyl)and $\gamma$-(1-naphthyl)-butyric acids by Nocardia $s p$. strain $\boldsymbol{P}_{\mathbf{2}}$

The result of a manometric experiment suggested that the fatty acid sidechains of phenyl- and indolyl-butyric acids are oxidized more rapidly than that of $\gamma$-(1-naphthyl)-butyric acid. This was confirmed in an incubation experiment with these compounds set up in separate flasks with equal samples from the same washed suspension of strain $\mathrm{P}_{2}$ organisms. Examination of $24 \mathrm{hr}$. samples revealed that whereas the phenyl- and indolyl-butyric acids had been completely converted to their corresponding acetate derivative the $\gamma$-(1-naphthyl)-butyrate remained practically unchanged. In another experiment with phenyl-and indolyl-butyric acids in which samples were examined at shorter intervals it was found that in $3 \mathrm{hr}$. there had been complete conversion of phenylbutyric acid, whereas after $7 \mathrm{hr}$. indolylbutyric acid was still only c. $50 \%$ converted. 


\section{Occurrence of $\beta$-hydroxy-and $\alpha \beta$-unsaturated intermediates during $\beta$-oxidation by strain $\boldsymbol{T}_{\mathbf{1 6}}$ and $\boldsymbol{P}_{\mathbf{2}}$}

With the techniques used in an earlier study of the $\beta$-oxidation of $\beta$-phenylpropionic acid to benzoic acid it was shown that cinnamic acid was an intermediate (Webley et al. 1955), but as small amounts of the $\beta$-hydroxy intermediate would probably not have been detected, this system has been reinvestigated. Samples were taken for analysis at 8,24 , and $48 \mathrm{hr}$. after addition of strain $\mathrm{P}_{2}$ organisms to $0.02 \mathrm{M}$-phenylpropionate. The infrared spectra (dioxan solution) of the mixture of acids extracted from samples showing partial conversion to benzoic acid ( 8 and $24 \mathrm{hr}$.) had no detectable absorption arising from alcoholic hydroxyl groups, indicating that the $\beta$-hydroxy intermediate, if present, did not exceed $10 \%$. Cinnamic acid (about $10 \%$ ) was clearly in evidence $\left(\mathrm{C}=\mathrm{C}\right.$ absorption at $\left.1640 \mathrm{~cm} .^{-1}\right)$. These observations were confirmed with $T_{16}$ organisms in a similar experiment. In contrast, during the $\beta$-oxidation of the variously substituted phenoxybutyric acids, the $\beta$-hydroxy acid intermediates have been detected in amounts between 25 and $75 \%$ of the total acids, but no $\alpha \beta$-unsaturated intermediates are detectable (i.e. less than $5 \%$ present). Neither $\beta$-hydroxy nor $\alpha \beta$-unsaturated acids have been found during $\beta$-oxidation of phenylbutyrate by $\mathbf{P}_{\mathbf{2}}$.

\section{DISCUSSION}

The following factors affect the $\beta$-oxidation of the fatty acid side-chain of $\omega$-aryl- and $\omega$-aryloxy- $n$-alkylcarboxylic acids by washed suspensions of the nocardias used in the present work. (a) An oxygen bridge between the fatty acid and the ring; as with phenylbutyric and phenoxybutyric and with $\gamma$-(1-naphthyl)butyric and $\gamma$-(1-naphthyloxy)butyric acids. (b) Ring substitution, particularly in the 2-position. (c) Nature of the ring substituents (e.g. chlorine and/or methyl groups). (d) Kind of ring structure to which the fatty acid is attached (phenyl, indolyl and naphthyl). (e) Position of attachment of the fatty acid; as with $\omega$-(1-naphthyloxy)- and $\omega$-(2-naphthyloxy)-propionic and -butyric acids. The effects of $(a),(b)$ and $(c)$ are most marked when the side-chain is short as in propionic or butyric acids, and although not tested this is probably true of $(d)$ and $(e)$.

A simple theory based on the steric and electronic (inductive) effects of the substituents can only give a partial explanation for all the observations in the present work. It is now clear that the appearance of $\beta$-hydroxy acid intermediates is common to all the $\omega$-aryloxybutyric acids, and is not solely an effect of chloro-substituents as previously suggested in work on 3- and 4-chlorophenoxybutyric acids (Webley et al. 1957). Our present work, however, repeatedly shows that the presence of a chlorine substituent appears to delay the conversion of the $\beta$-hydroxy acid intermediate to the acetic acid derivative leading to a greater accumulation of the former. This may be due to an inductive effect of the chlorine atom reinforcing the tendency of the ether oxygen to withdraw electrons from the side-chain. Further, while steric hindrance 
may be adequate to account for the slower oxidation of $\beta$-(1-naphthyl)propionic (Webley et al. 1956), and $\gamma$-(1-naphthyl)-butyric acid compared with the corresponding phenyl derivatives, it seems inadequate to account for the big difference in the rates of $\beta$-oxidation of $\gamma$-(1-naphthyloxy)- and $\gamma$-(2naphthyloxy)-butyric acids. Clearly work on isolated enzymes (cf. Stern \& del Campillo, 1955) involved in the various stages would be necessary to provide fuller explanations. The results obtained with MCPB and 2:4-DB are of interest since these compounds are well-known herbicides; the principle on which they operate has been described by Wain (1955) who suggested that two different plants might respond differently to these compounds because the $\beta$-oxidizing enzymes were unable to operate in the unaffected plant. The present work suggests, by analogy, that the $\beta$-oxidizing system of the unaffected plant may be affected to a greater extent by substitution than that of the sensitive plant.

We wish to thank Professor R. L. Wain (Wye College) and Dr B. J. Heywood (May and Bakers Ltd.) for the specimens of $\omega$-substituted phenoxy acids used, Dr R. J. Byrde and Dr D. Woodcock (Long Ashton Research Station) for samples of $\omega$-(1-naphthyloxy)- and $\omega$-(2-naphthyloxy)-butyric and -propionic acids, Professor M. Julia (University of Paris) for the ester of $\beta$-hydroxy- $\gamma$-(2-naphthyloxy)-butyric acid and Dr N. Walker (Rothamsted Experimental Station) for a specimen of 4-chlorocatechol.

\section{REFERENCES}

Bellamy, L. T., Thomas, L. C. \& Williams, R. L. (1956). Infrared spectra and polar effects. IV. Steric restrictions of polar effects and their applications in studies on rotational isomerism. J. chem. Soc. p. 3704.

Byrde, R. J. W., Harris, J. F. \& Wooncock, D. (1956). Fungal detoxication. The metabolism of $\omega$-(2-naphthyloxy)- $n$-alkylcarboxylic acids by Aspergillus niger. Biochem. J. 64, 154.

Fawcett, C. H., Ingram, J. M. A. \& WaIn, R. L. (1954). The $\beta$-oxidation of $\omega$-phenylalkylcarboxylic acids in the flax plant in relation to their plant growth regulating activity. Proc. Roy. Soc. B, 142, 60.

Fewster, M. E. \& HaLL, D. A. (1951). Application of buffered solvent systems to the detection of aromatic acids by paper partition chromatography. Nature, Lond. $168,78$.

Grün, A. (1925). Analyse der Fette und Wachse, 1, 144. Berlin: Springer.

JeAnes, A., Wise, C. S. \& Dimler, R. J. (1951). Improved techniques in paper chromatography of carbohydrates. Analyt. Chem. 23, 415.

Lynen, F. (1953). Functional group of co-enzyme $A$ and its metabolic relations, especially in the fatty acid cycle. Fed. Proc. $12,683$.

Mahler, H. R. (1953). Role of co-enzyme $A$ in fatty acid metabolism Fed. Proc. $12,694$.

Stern, J. R. \& Del Campillo, A. (1955). Enzymes of fatty acid metabolism (II). Properties of crystalline crotonase. J. biol. Chem. 218, 985.

WAIN, R. L. (1955). A new principle of weed control. Agric. Rev. 1, 25.

WaIn, R. L. (1956). The regulation of plant growth with chemicals. Sci. Progr. 44, 604.

Webley, D. M. (1954). The morphology of Nocardia opaca, Waksman and Henrici (Proactinomyces opacus Jensen) when grown on hydrocarbons, vegetable oils, fatty acids and related substances. J. gen. Microbiol. 11, 420 . 
Webley, D. M., DufF, R. B. \& FArmer, V. C. (1955). Beta-oxidation of fatty acids by Nocardia opaca. J. gen. Microbiol. 13, 361.

Webley, D. M., DuFf, R. B. \& FArmer, V. C. (1956). Evidence for $\beta$-oxidation in the metabolism of saturated aliphatic hydrocarbons by soil species of Nocardia. Nature, Lond. 178, 1467.

Webley, D. M., DuFF, R. B. \& FARMer, V. C. (1957). Formation of a $\beta$-hydroxy acid as an intermediate in the microbiological conversion of monochlorophenoxybutyric acids to the corresponding substituted acetic acids. Nature, Lond. 179, 1130.

(Received 30 December 1957) 